\title{
Article
}

\section{On properties of inner product type integral transformers}

\section{Benard Okelo}

Department of Pure and Applied Mathematics, Jaramogi Oginga Odinga University of Science and Technology, Box 210-40601, Bondo-Kenya.; bnyaare@yahoo.com

Received: 5 October 2020; Accepted: 14 December 2020; Published: 23 December 2020.

\begin{abstract}
In this paper, we give characterizations of certain properties of inner product type integral transformers. We first consider unitarily invariant norms and operator valued functions. We then give results on norm inequalities for inner product type integral transformers in terms of Landau inequality, Grüss inequality. Lastly, we explore some of the applications in quantum theory.
\end{abstract}

Keywords: Norm inequality, unitarily invariant norm, operator valued function, norm ideal, inner product type integral transformer.

MSC: 46B20, 47L05.

\section{Introduction}

$\mathbf{L}$ et $\mathcal{H}$ be an infinite dimensional complex Hilbert space and $\mathcal{B}(\mathcal{H})$ be the algebra of all bounded linear operators on $\mathcal{H}$. In this paper, we discuss various types of norm inequalities for inner product type integral transformers in terms of Landau type inequality, Grüss type inequality and Cauchy-Schwarz type inequality. We shall also consider the applications in quantum theory. We begin by the following definition:

Definition 1. Grüss inequality states that if $f$ and $g$ are integrable real functions on $[a, b]$ such that $C \leq f(x) \leq$ $D$ and $E \leq g(x) \leq F$ hold for some real constants $C, D, E, F$ and for all $x \in[a, b]$, then

$$
\left|\frac{1}{b-a} \int_{a}^{b} f(x) g(x) d x-\frac{1}{(b-a)^{2}} \int_{a}^{b} f(x) d x \int_{a}^{b} g(x) d x\right| \leq \frac{1}{4}(D-C)(F-E) .
$$

Inequality (1) is very interesting to many researchers and it has been considered in many studies whereby conditions on functions are varied to give different estimates (see [1] and references therein). More on this inequality (and the classical one [2]) are discussed in the sequel.

Next, we discuss a very important definition of inner product type integral (i.p.t.i) transformer which is key to our study.

Definition 2. Consider weakly $\mu^{*}$-measurable operator valued (o.v) functions $A, B: \Omega \rightarrow \mathcal{B}(\mathcal{H})$ and for all $X \in \mathcal{B}(\mathcal{H})$. Let the function $t \rightarrow A_{t} X B_{t}$ be also weakly $\mu^{*}$-measurable. If these functions are Gel'fand integrable for all $X \in \mathcal{B}(\mathcal{H})$, then the inner product type linear transformation $X \rightarrow \int_{\Omega} A_{t} X B_{t} d t$ is called an inner product type integral (i.p.t.i) transformer on $\mathcal{B}(\mathcal{H})$ and denoted by $\int_{\Omega} A_{t} \otimes B_{t} d t$ or $\mathcal{I}_{A, B}$.

Remark 1. If $\mu$ is the counting measure on $\mathbb{N}$ then such transformers are known as elementary operators whose certain properties have been studied in details (see [3] and the references therein).

\section{Preliminaries}

In this section, we consider a special type of norms called the unitarily invariant norm. We give its description in details which will be useful in the sequel. Let $\mathcal{C}_{\infty}(\mathcal{H})$ denote the space of all compact linear operators acting on a separable, complex Hilbert space $\mathcal{H}$. Each symmetric gauge function $\Phi$, denoted by (s.g.), on sequences gives rise to a unitarily invariant $(u . i)$ norm on operators defined by $\|X\|_{\Phi}=\Phi\left(\left\{s_{n}(X)\right\}_{n=1}^{\infty}\right)$ with $s_{1}(X) \geq s_{2}(X) \geq \ldots$ being the singular values of $X$, i.e., the eigenvalues of $|X|=\left(X^{*} X\right)^{\frac{1}{2}}$. We denote 
any such norm by the symbol $\||\cdot|\| \mid$, which is therefore defined on a naturally associated norm ideal $\mathcal{C}_{\|\| \cdot \mid \|}(\mathcal{H})$ of $\mathcal{C}_{\infty}(\mathcal{H})$ and satisfies the invariance property $\left|\left\|U X V\left|\left\|=\left|\|X \mid\|\right.\right.\right.\right.\right.$ for all $X \in \mathcal{C}_{\||\cdot|\| \mid}(\mathcal{H})$ and for all unitary operators $U, V \in \mathcal{B}(\mathcal{H})$. One of the well known among $u$.i. norms are the Schatten $p$-norms defined for $1 \leq p<\infty$ as $\|X\|_{p}=\sqrt[p]{\sum_{n=1}^{\infty} s_{n}^{p}(X)}$, while $\|X\|_{\infty}=\|X\|=s_{1}(X)$ coincides with the operator norm $\|X\|$. Minimal and maximal $u$.i. norm are among Schatten norms, i.e., $\|X\|_{\infty} \leq\|\| X\|\mid \leq\| X \|_{1}$ for all $X \in \mathcal{C}_{1}(\mathcal{H}$ ) (see inequality (IV.38) [4]). For $f, g \in \mathcal{H}$, we will denote by $g^{*} \otimes f$ one dimensional operator $\left(g^{*} \otimes f\right) h=\langle h, g\rangle f$ for all $h \in \mathcal{H}$ and it is known that the linear span of $\left\{g^{*} \otimes f \mid f, g \in \mathcal{H}\right\}$ is dense in each of $\mathcal{C}_{p}(\mathcal{H})$ for $1 \leq p \leq \infty$. Schatten $p$-norms are also classical examples of $p$-reconvexized norms. Namely, any $u$.i. norm $\|.\|_{\Phi}$ could be $p$-reconvexized for any $p \geq 1$ by setting $\|A\|_{\Phi^{(p)}}=\left\||A|^{p}\right\|_{\Phi}^{\frac{1}{p}}$ for all $A \in \mathcal{B}(\mathcal{H})$ such that $|A|^{p} \in \Phi(\mathcal{H})$. For the proof of the triangle inequality and other properties of these norms, see [2] and for the characterization of the dual norm for $p$-reconvexized, see Theorem 2.1 [2].

The set $\mathcal{C}_{\| \cdot|\cdot|}=\{A \in \mathcal{K}(\mathcal{H}):\|\| A \| \mid<\infty\}$ is a closed self-adjoint ideal $\mathcal{J}$ of $\mathcal{B}(\mathcal{H})$ containing finite rank operators. It enjoys the following properties. First, for all $A, B \in \mathcal{B}(\mathcal{H})$ and $X \in \mathcal{J},\|\| A X B \| \mid \leq$ $\|A\||\|X|\|\mid\| B \|$. Secondly, if $X$ is a rank one operator, then $\||X|\|=\|X\|$. The Ky Fan norm as an example of unitarily invariant norms is defined by $\|A\|_{(k)}=\sum_{j=1}^{k} s_{j}(A)$ for $k=1,2, \ldots$. The Ky Fan dominance Theorem [5] states that $\|A\|_{(k)} \leq\|B\|_{(k)}(k=1,2, \ldots)$ if and only if $\||A\||| \leq|| B \mid\|$ for all unitarily invariant norms $\||\cdot|\|$, see [6] for more information on unitarily invariant norms. The inequalities involving unitarily invariant norms have been of special interest (see [5] and the references therein).

Lemma 1. Let $\mathcal{T}$ and $\mathcal{S}$ be linear mappings defined on $\mathcal{C}_{\infty}(\mathcal{H})$. If $\|\mathcal{T} X\| \leq\|\mathcal{S} X\|$ for all $X \in \mathcal{C}_{\infty}(\mathcal{H}),\|\mathcal{T} X\|_{1} \leq$ $\|\mathcal{S} X\|_{1}$ for all $X \in \mathcal{C}_{\infty}(\mathcal{H})$, then $\mathcal{T} X \leq \mathcal{S} X$ for all unitarily invariant norms.

Proof. The norms $\|\cdot\|$ and $\|\cdot\|_{1}$ are dual to each other in the sense that $\|X\|=\sup _{\|Y\|_{1}=1}|\operatorname{tr}(X Y)|$ and $\|X\|_{1}=\sup _{\|Y\|=1}|\operatorname{tr}(X Y)|$. Hence $\left\|\mathcal{T}^{*} X\right\| \leq\left\|\mathcal{S}^{*} X\right\|$ and $\left\|\mathcal{T}^{*} X\right\|_{1} \leq\left\|\mathcal{S}^{*} X\right\|_{1}$. Consider the Ky Fan norm $\|\cdot\|_{(k)}$. Its dual norm is $\|\cdot\|_{(k)}^{\sharp}=\max \left\{\|\cdot\|,(1 / k)\|\cdot\|_{1}\right\}$. Thus, by duality, $\|\mathcal{T} X\|_{(k)} \leq\|\mathcal{S} X\|_{(k)}$ and the result follows by Ky Fan dominance property [6].

An operator $A \in \mathcal{B}(\mathcal{H})$ is called $G_{1}$ operator if the growth condition

$$
\left\|(z-A)^{-1}\right\|=\frac{1}{\operatorname{dist}(z, \sigma(A))}
$$

holds for all $z$ not in the spectrum $\sigma(A)$ of $A$. Here $\operatorname{dist}(z, \sigma(A))$ denotes the distance between $z$ and $\sigma(A)$. It is known that hyponormal (in particular, normal) operators are $G_{1}$ operators [4].

Let $A, B \in \mathcal{B}(\mathcal{H})$ and let $f$ be a function which is analytic on an open neighborhood $\Omega$ of $\sigma(A)$ in the complex plane. Then $f(A)$ denotes the operator defined on $\mathcal{H}$ by $f(A)=\frac{1}{2 \pi i} \int_{C} f(z)(z-A)^{-1} d z$, called the Riesz-Dunford integral, where $C$ is a positively oriented simple closed rectifiable contour surrounding $\sigma(A)$ in $\Omega$ (see [2] and the references therein). The spectral mapping theorem asserts that $\sigma(f(A))=f(\sigma(A))$. Throughout this paper, $\mathbb{D}=\{z \in \mathbb{C}:|z|<1\}$ denotes the unit disk, $\partial \mathbb{D}$ stands for the boundary of $\mathbb{D}$ and $d_{A}=$ $\operatorname{dist}(\partial \mathbb{D}, \sigma(A))$. In addition, we adopt the notation $\mathfrak{H}=\{f: \mathbb{D} \rightarrow \mathbb{C}: f$ is analytic, $\Re(f)>0$ and $f(0)=1\}$.

In this work, we present some upper bounds for $\|f f(A) X g(B) \pm X \mid\|$, where $A, B$ are $G_{1}$ operators, $\||\cdot|\|$ is a unitarily invariant norm and $f, g \in \mathfrak{H}$. Further, we find some new upper bounds for the the Schatten 2-norm of $f(A) X \pm X g(B)$. Up-to this juncture, we find some upper estimates for $\|f f(A) X g(B)+X\| \|$ in terms of ||||$A X B|+| X||||$ and ||$|f(A) X g(B)-X|||$ in terms of ||||$A X|+| X B||||$, where $A, B$ are $G_{1}$ operators and $f, g \in \mathcal{H}$.

Proposition 1. If $A, B \in \mathcal{B}(\mathcal{H})$ are $G_{1}$ operators with $\sigma(A) \cup \sigma(B) \subset \mathbb{D}$ and $f, g \in \mathcal{H}$, then for every $X \in \mathcal{B}(\mathcal{H})$ and for every unitarily invariant norm ||$|\cdot|||$, the inequality ||$|f(A) X g(B)+X||| \leq \frac{2 \sqrt{2}}{d_{A} d_{B}}|||| A X B|+| X||||$ holds.

Proof. From the Herglotz representation Theorem [1], it follows that $f \in \mathcal{H}$ can be represented as 


$$
f(z)=\int_{0}^{2 \pi} \frac{e^{i \alpha}+z}{e^{i \alpha}-z} d \mu(\alpha)+i \Im f(0)=\int_{0}^{2 \pi} \frac{e^{i \alpha}+z}{e^{i \alpha}-z} d \mu(\alpha),
$$

where $\mu$ is a positive Borel measure on the interval $[0,2 \pi]$ with finite total mass $\int_{0}^{2 \pi} d \mu(\alpha)=f(0)=1$. Similarly $g(z)=\int_{0}^{2 \pi} \frac{e^{i \alpha}+z}{e^{i \alpha}-z} d v(\alpha)$ for some positive Borel measure $v$ on the interval $[0,2 \pi]$ with finite total mass 1 . We have

$$
f(A) X g(B)+X=\int_{0}^{2 \pi} \int_{0}^{2 \pi}\left[\left(e^{i \alpha}-A\right)^{-1}\left(e^{i \alpha}+A\right) X\left(e^{i \beta}+B\right)\left(e^{i \beta}-B\right)^{-1}+X\right] d \mu(\alpha) d v(\beta) .
$$

By some computation, we have

$$
\|\| f(A) X g(B)+X \mid\left\|\leq \int_{0}^{2 \pi} \int_{0}^{2 \pi} 2\right\|\left(e^{i \alpha}-A\right)^{-1}\|\|\left\|A X B+e^{i \alpha} X e^{i \beta}\right\|\|\|\left(e^{i \alpha}-B\right)^{-1} \| d \mu(\alpha) d v(\beta) .
$$

Since $A$ and $B$ are $G_{1}$ operators, we deduce that

$$
\left\|\left(e^{i \alpha}-A\right)^{-1}\right\|=\frac{1}{\operatorname{dist}\left(e^{i \alpha}, \sigma(A)\right)} \leq \frac{1}{\operatorname{dist}(\partial \mathbb{D}, \sigma(A))}=\frac{1}{d_{A}},
$$

and similarly $\left\|\left(e^{i \beta}-B\right)^{-1}\right\| \leq \frac{1}{d_{B}}$. Now, for every positive operators $C, D$, every non-negative operator monotone function $h(t)$ on $[0, \infty)$ and every unitarily invariant norm $\||\cdot|\|$, we have $\|\mid h(A+B)\| \| \leq$ $\|h(A)+h(B) \mid\|$. Now, from the Ky Fan dominance theorem, we infer that

$$
\left\|\left|A X B+e^{i \alpha} X e^{i \beta}\right|\right\| \leq \sqrt{2}|||| A X B|+| X|||| .
$$

Therefore, it follows from inequality (3) and Equation (4) that

$$
|||f(A) X g(B)+X||| \leq \frac{2 \sqrt{2}}{d_{A} d_{B}}|||| A X B|+| X||||,
$$

which completes the proof.

Theorem 1. Let $f, g \in \mathcal{H}$ and $A \in \mathcal{B}(\mathcal{H})$ be a $G_{1}$ operator with $\sigma(A) \subset \mathbb{D}$. The inequality $\left\|\left|f(A) X g\left(A^{*}\right)+X\right|\right\| \leq$ $\frac{2}{d_{A}^{2}}|||A| X\left|A^{*}+\right| X||||$ holds for every normal operator $X \in \mathcal{B}(\mathcal{H})$ commuting with $A$ and for every unitarily invariant norm $|\| \cdot|||$.

Proof. Let $X$ and $A X B$ be normal. Since ||$|C+D|\|\leq|\|||C|+|D||| \mid$ for any normal operators $C$ and $D$, the constant $\sqrt{2}$ can be reduced to 1 in Equation (4). Now from Fuglede-Putnam theorem, if $A \in \mathcal{B}(\mathcal{H})$ is an operator, $X \in(B)((H))$ is normal and $A X=X A$, then $A X^{*}=X^{*} A$. Thus if $X$ is a normal operator commuting with a $G_{1}$ operator $A$, then $A X A^{*}$ is normal, $\left|A X A^{*}\right|=A|X| A^{*}$ and $A^{*}$ is a $G_{1}$ operator with $d_{A^{*}}=d_{A}$. By Proposition 1 the proof is complete.

Next, letting $A=B$ in Proposition 1, we obtain the following result.

Corollary 1. Let $f, g \in \mathcal{H}$ and $A \in \mathcal{B}(\mathcal{H})$ be a $G_{1}$ operator with $\sigma(A) \subset \mathbb{D}$. Then $\||f(A) X g(A)-X|\| \leq$ $\frac{2 \sqrt{2}}{d_{A}^{2}}|||| A X|+| X A||||$ for every $X \in \mathcal{B}(\mathcal{H})$ and for every unitarily invariant norm ||$|\cdot|||$.

Setting $X=I$ in Proposition 1 again, we obtain the following result. 
Corollary 2. Let $f, g \in \mathfrak{H}$ and $A, B \in \mathbb{M}_{n}$ be $G_{1}$ matrices such that $\sigma(A) \cup \sigma(B) \subset \mathbb{D}$. Then $\||| f(A) g(B)+I \mid\| \leq$ $\frac{2 \sqrt{2}}{d_{A} d_{B}}|||| A B|+I|||$ for every unitarily invariant norm $\||\cdot|||$.

Corollary 3. If $A \in \mathcal{B}(\mathcal{H})$ is self-adjoint and $f$ is a continuous complex function on $\sigma(A)$, then $f\left(U A U^{*}\right)=$ $U f(A) U^{*}$ for all unitaries $U$.

Proof. By the Stone-Weierstrass theorem, there is a sequence $\left(p_{n}\right)$ of polynomials uniformly converging to $f$ on $\sigma(A)$. Hence,

$$
f\left(U A U^{*}\right)=\lim _{n} p_{n}\left(U A U^{*}\right)=U\left(\lim _{n} p_{n}(A)\right) U^{*}=U f(A) U^{*} .
$$

We note that $\sigma\left(U A U^{*}\right)=\sigma(A)$.

We conclude this section by presenting some inequalities involving the Hilbert-Schmidt norm $\|\cdot\|_{2}$.

Theorem 2. Let $A, B \in \mathbb{M}_{n}$ be Hermitian matrices satisfying $\sigma(A) \cup \sigma(B) \subset \mathbb{D}$ and let $f, g \in \mathfrak{H}$. Then $\| f(A) X \pm$ $X g(B)\left\|_{2} \leq\right\| \frac{X+|A| X}{d_{A}}+\frac{X+X|B|}{d_{B}} \|_{2}$.

Proof. Let $A=U D\left(v_{j}\right) U^{*}$ and $B=V D\left(\mu_{k}\right) V^{*}$ be the spectral decomposition of $A$ and $B$ and let $Y=U^{*} X V:=$ $\left[y_{j k}\right]$. Noting that $\left|e^{i \alpha}-\lambda_{j}\right| \geq d_{A}$ and $\left|e^{i \beta}-\mu_{k}\right| \geq d_{B}$, we have from [7] that

$$
\begin{aligned}
\|f(A) X \pm X g(B)\|_{2}^{2} & =\sum_{j, k}\left|f\left(\lambda_{j}\right) \pm g\left(\mu_{k}\right)\right|^{2}\left|y_{j k}\right|^{2} \\
& \leq \sum_{j, k}\left(\frac{1+\left|\lambda_{j}\right|}{d_{A}}+\frac{1+\left|\mu_{k}\right|}{d_{B}}\right)^{2}\left|y_{j k}\right|^{2} \\
& =\left\|\frac{X+|A| X}{d_{A}}+\frac{X+X|B|}{d_{B}}\right\|_{2}^{\prime}
\end{aligned}
$$

which completes the proof.

\section{Operators in function spaces}

In this section, we present some results on operator valued functions. From [5], if $(\Omega, \mathcal{M}, \mu)$ is a measure space, for a $\sigma$-finite measure $\mu$ on $\mathcal{M}$, the mapping $\mathcal{A}: \Omega \rightarrow \mathcal{B}(\mathcal{H})$ will be called $[\mu]$ weakly*-measurable if a scalar valued function $t \rightarrow \operatorname{tr}\left(A_{t} Y\right)$ is measurable for any $Y \in \mathcal{C}_{1}(\mathcal{H})$. Moreover, if all these functions are in $\iota^{1}(\Omega, \mu)$, then since $\mathcal{B}(\mathcal{H})$ is the dual space of $\mathcal{C}_{1}(\mathcal{H})$, for any $E \in \mathcal{M}$, we have the unique operator $I_{E} \in \mathcal{B}(\mathcal{H})$, called the Gel'fand or weak *-integral of $\mathcal{A}$ over $E$, such that

$$
\operatorname{tr}\left(\mathcal{I}_{E} Y\right)=\int_{E} \operatorname{tr}\left(A_{t} Y\right) d t \quad \text { for all } Y \in \mathcal{C}_{\infty}(\mathcal{H})
$$

We denote it by $\int_{E} A_{t} d \mu(t)$ or $\int_{E} A d \mu$. We consider the following important aspect.

Proposition 2. $A: \Omega \rightarrow \mathcal{B}(\mathcal{H})$ is $[\mu]$ if and only if scalar valued functions $t \rightarrow\left\langle A_{t} f, f\right\rangle$ are $[\mu]$ measurable (resp. integrable) for every $f \in \mathcal{H}$.

Proof. Every one dimensional operator $f^{*} \otimes f$ is in $C_{1}(H)$ and $\operatorname{tr}\left(A_{t}\left(f^{*} \otimes f\right)\right)=\operatorname{tr}\left(f^{*} \otimes A_{t} f\right)=\left\langle A_{t} f, f\right\rangle$, so that $[\mu]$ weak *-measurability (resp. $[\mu]$ weak *-integrability) of $A$ directly implies measurability (resp. integrability) of $\left\langle A_{t} f, f\right\rangle$ for any $f \in \mathcal{H}$. The converse follows immediately from [4] and this completes the proof.

We note that in view of Proposition 2, the Equation (5) of Gel'fand integral for o.v. functions can be reformulated as follows [2]:

Proposition 3. If $\langle A f, f\rangle \in L^{1}(E, \mu)$ for all $f \in \mathcal{H}$, for some $E \in \mathcal{M}$ and a $\mathcal{B}(\mathcal{H})$-valued function $A$ on $E$, then the mapping $f \rightarrow \int_{E}\left\langle A_{t} f, f\right\rangle d \mu(t)$ represents a quadratic form of bounded operator $\int_{E} A d m$ or $\int_{E} A_{t} d \mu(t)$, satisfying the following $\left\langle\left(\int_{E} A_{t} d \mu(t)\right) f, g\right\rangle=\int_{E}\langle A t f, g\rangle d \mu(t)$, for all $f, g \in \mathcal{H}$. 
Proof. It suffices to show that for all $E \in \mathcal{M}, \Phi_{E}(f, g)=\int_{E}\left\langle A_{t} f, g\right\rangle d \mu(t)$, for all $f, g \in \mathcal{H}$, defines a bounded sesquilinear functional $\Phi$ on $\mathcal{H}$. Indeed, by [1], we have $\left|\Phi_{E}(f, g)\right| \leq \int_{E}\left|\left\langle A_{t} f, g\right\rangle\right| d \mu(t) \leq\left\|A_{t} f, g\right\|_{L^{1}} \leq$ $M\|f\|\|g\|$ for all $f, g \in \mathcal{H}$ since integration is a contractive functional on $七^{1}(\Omega, \mu)$. This completes the proof.

Remark 2. It is known from [1] that for a $[\mu] A: \Omega \rightarrow \mathcal{B}(\mathcal{H})$ we have that $A^{*} A$ is Gel'fand integrable if and only if $\int_{\Omega}\left\|A_{t} f\right\|^{2} d \mu(t)<\infty$, for all $f \in \mathcal{H}$. Moreover, for a $[\mu]$ function $A: \Omega \rightarrow \mathcal{B}(\mathcal{H})$. Let us consider a linear transformation $\vec{A}: D_{\vec{A}} \rightarrow L^{2}(\Omega, \mu, \mathcal{H})$, with the domain $D_{\vec{A}}=\left\{f \in \mathcal{H} \mid \int_{\Omega}\left\|A_{t} f\right\|^{2} d \mu(t)<\infty\right\}$, defined by $(\vec{A} f)(t)=A_{t} f$. and all $f \in D_{\vec{A}}$.

In the next section, we devote our efforts to results on inner product type integral transformers in terms of Landau, Cauchy-Schwarz and Grüss type norm inequalities.

\section{Norm inequalities}

In this section, we consider various types of norm inequalities for inner product type integral transformers discussed in [1,2,4,7]. From [1], a sufficient condition is provided when $A^{*}$ and $B$ from Definition 2 are both in $L_{G}^{2}(\Omega, d \mu, \mathcal{B}(\mathcal{H}))$. If each of families $\left(A_{t}\right)_{t \in \Omega}$ and $\left(B_{t}\right)_{t \in \Omega}$ consists of commuting normal operators, then by Theorem 3.2 [1], the i.p.t.i transformer $\int_{\Omega} A_{t} \otimes B_{t} d \mu(t)$ leaves every $u$.i. norm ideal $\mathcal{C}_{|\|\cdot \mid\|}(\mathcal{H})$ invariant and the following Cauchy-Schwarz inequality holds:

$$
\left\|\int _ { \Omega } A _ { t } X B _ { t } d \mu ( t ) \left|\left\|\leq\left|\left\|\sqrt{\int_{\Omega} A_{t}^{*} A_{t}} d \mu(t) \sqrt{\int_{\Omega} B_{t}^{*} B_{t} d \mu(t)} \mid\right\|,\right.\right.\right.\right.
$$

for all $X \in \mathcal{C}_{|\|\cdot \mid\|}(\mathcal{H})$. Normality and commutativity condition can be dropped for Schatten $p$-norms as shown in Theorem 3.3 [1]. In Theorem 3.1 [2], a formula for the exact norm of the i.p.t.i transformer $\int_{\Omega} A_{t} \otimes B_{t} d \mu(t)$ acting on $\mathcal{C}_{2}(\mathcal{H})$ is found. In Theorem 2.1 [2], the exact norm of the i.p.t.i transformer $\int_{\Omega} A_{t}^{*} \otimes A_{t} d \mu(t)$ is given for two specific cases:

$$
\begin{aligned}
\left\|\int_{\Omega} A_{t}^{*} \otimes A_{t} d \mu(t)\right\|_{B(H) \rightarrow \mathcal{C}_{\Phi}(\mathcal{H})} & =\left\|\int_{\Omega} A_{t}^{*} A_{t} d \mu(t)\right\|_{\mathcal{C}_{\Phi}(\mathcal{H})}, \\
\left\|\int_{\Omega} A_{t}^{*} \otimes A_{t} d \mu(t)\right\|_{\mathcal{C}_{\Phi}(\mathcal{H}) \rightarrow \mathcal{C}_{1}(\mathcal{H})} & =\left\|\int_{\Omega} A_{t} A_{t}^{*} d \mu(t)\right\|_{\mathcal{C}_{\Phi_{*}}(\mathcal{H})},
\end{aligned}
$$

where $\Phi_{*}$ stands for a s.g. function related to the dual space $\left(\mathcal{C}_{\Phi}(\mathcal{H})\right)^{*}$. The norm appearing in (7) and its associated space $L_{G}^{2}\left(\Omega, d \mu, \mathcal{B}(\mathcal{H}), \mathcal{C}_{\Phi}(\mathcal{H})\right)$ present only a special case of norming a field $A=\left(A_{t}\right)_{t \in \Omega}$. A much wider class of norms $\|\cdot\|_{\Phi, \Psi}$ and their associated spaces $L_{G}^{2}\left(\Omega, d \mu, \mathcal{B}(\mathcal{H}), \mathcal{C}_{\Phi}(\mathcal{H})\right)$ are given by [2]:

$$
\|A\|_{\Phi, \Psi}=\left\|\int_{\Omega} A_{t}^{*} \otimes A_{t} d \mu(t)\right\|_{B\left(\mathcal{C}_{\Phi}(\mathcal{H}), \mathcal{C}_{\Psi}(\mathcal{H})\right)}^{\frac{1}{2}}
$$

for an arbitrary pair of s.g. functions $\Phi$ and $\Psi$. For the proof of completeness of the space $L_{G}^{2}\left(\Omega, d \mu, \mathcal{C}_{\Phi}(\mathcal{H}), \mathcal{C}_{\Psi}(\mathcal{H})\right)$, see Theorem 2.2 [2]. Before going into the details of this section lets consider the following Proposition which will be useful in the sequel [7]. We give its proof for completion.

Proposition 4. Let $\mu$ be a probability measure on $\Omega$, then for every field $\left(\mathcal{A}_{t}\right)_{t \in \Omega}$ in $L^{2}(\Omega, \mu, \mathcal{B}(\mathcal{H})$ ), for all $B \in \mathcal{B}(\mathcal{H})$, for all unitarily invariant norms ||$|\cdot|||$ and for all $\theta>0$,

$$
\begin{aligned}
\int_{\Omega}\left|\mathcal{A}_{t}-B\right|^{2} d \mu(t) & =\int_{\Omega}\left|\mathcal{A}_{t}-\int_{\Omega} A_{t} d \mu(t)\right|^{2} d \mu(t)+\left|\int_{\Omega} A_{t} d \mu(t)-B\right|^{2} \\
& \geq \int_{\Omega}\left|\mathcal{A}_{t}-\int_{\Omega} A_{t} d \mu(t)\right|^{2} d \mu(t)=\int_{\Omega}\left|\mathcal{A}_{t}\right|^{2} d \mu(t)-\left|\int_{\Omega} A_{t} d \mu(t)\right|^{2}
\end{aligned}
$$




$$
\begin{aligned}
\min _{B \in \mathcal{B}(\mathcal{H})}\left|\left\|\left|\int_{\Omega}\right| \mathcal{A}_{t}-\left.B\right|^{2} d \mu(t)||^{\theta} \mid\right\|\right. & =\left|\left\|\left|\int_{\Omega}\right| \mathcal{A}_{t}-\left.\left.\int_{\Omega} A_{t} d \mu(t)\right|^{2} d \mu(t)\right|^{\theta}\right\|\right| \mid \\
& =\left.\left|\int_{\Omega}\right| \mathcal{A}_{t}\right|^{2} d \mu(t)-\left|\int_{\Omega} A_{t} d \mu(t)\right|^{2} \|\left.\right|^{\theta}||
\end{aligned}
$$

Thus, the considered minimum is always obtained for $B=\int_{\Omega} A_{t} d \mu(t)$.

Proof. The expression (9) is trivial and the inequality (10) follows from (9), while identity (10) is just a a special case of Lemma 2.1 [1] applied for $k=1$ and $\delta_{1}=\Omega$.

As $0 \leq A \leq B$ for $A, B \in \mathcal{C}_{\infty}(\mathcal{H})$ implies $s_{n}^{\theta}(A) \leq s_{n}^{\theta}(B)$ for all $n \in \mathbb{N}$, as well as \|\|$A^{\theta}\left|\left\|\leq\left|\left\|B^{\theta} \mid\right\|\right.\right.\right.$, then (12) follows.

Recall that, for a pair of random real variables $(Y, Z)$, its coefficient of correlation

$$
\rho_{Y, Z}=\frac{|E(Y Z)-E(Y) E(Z)|}{\sigma(Y) \sigma(Z)}=\frac{|E(Y Z)-E(Y) E(Z)|}{\sqrt{E\left(Y^{2}\right)-E^{2}(Y)} \sqrt{E\left(Z^{2}\right)-E^{2}(Z)}}
$$

always satisfies $\left|\rho_{Y, Z}\right| \leq 1$. For square integrable functions $f$ and $g$ on $[0,1]$ and $D(f, g)=\int_{0}^{1} f(t) g(t) d t-$ $\int_{0}^{1} f(t) d t \int_{0}^{1} g(t) d t$. Landau proved that $|D(f, g)| \leq \sqrt{D(f, f) D(g, g)}$.

The following result represents a generalization of Landau inequality in $u . i$. norm ideals [2] for Gel'fand integrals of $0 . v$. functions with relative simplicity of its formulation.

Theorem 3. If $\mu$ is a probability measure on $\Omega$. Let both fields $\left(A_{t}\right)_{t \in \Omega}$ and $\left(B_{t}\right)_{t \in \Omega}$ be in $L^{2}(\Omega, \mu, \mathcal{B}(\mathcal{H}))$ consisting of commuting normal operators and consider

$$
\sqrt{\int_{\Omega}\left|A_{t}\right|^{2}-\left|\int_{\Omega} A_{t} d \mu(t)\right|^{2}} X \sqrt{\int_{\Omega}\left|B_{t}\right|^{2} d \mu(t)-\left|\int_{\Omega} B_{t} d \mu(t)\right|^{2}}
$$

for some $X \in B(H)$. Then

$$
\int_{\Omega} A_{t} X B_{t} d \mu(t)-\int_{\Omega} A_{t} d t X \int_{\Omega} B_{t} d \mu(t) \in C_{|\|\cdot \mid\|}(H)
$$

Proof. First, we have the following Korkine type identity for i.p.t.i transformers

$$
\begin{aligned}
\int_{\Omega} A_{t} X B_{t} d \mu(t)-\int_{\Omega} A_{t} d \mu(t) X \int_{\Omega} B_{t} d \mu(t) & =\int_{\Omega} d \mu(s) \int_{\Omega} A_{t} X B_{t} d \mu(t)-\int_{\Omega} \int_{\Omega} A_{t} X B_{s} d \mu(s) d \mu(t) \\
& =\frac{1}{2} \int_{\Omega^{2}}\left(A_{s}-A_{t}\right) X\left(B_{s}-B_{t}\right) d(\mu \times \mu)(s, t) .
\end{aligned}
$$

In this representation, we have $\left(A_{s}-A_{t}\right)_{(s, t) \in \Omega^{2}}$ and $\left(B_{s}-B_{t}\right)_{(s, t) \in \Omega^{2}}$ to be in $L^{2}\left(\Omega^{2}, \mu \times \mu, \mathcal{B}(\mathcal{H})\right)$ because by an application of the identity (13),

$$
\frac{1}{2} \int_{\Omega^{2}}\left|A_{s}-A_{t}\right|^{2} d(\mu \times \mu)(s, t)=\int_{\Omega}\left|A_{t}\right|^{2} d \mu(t)-\left|\int_{\Omega} A_{t} d \mu(t)\right|^{2}=\int_{\Omega}\left|A_{t}-\int_{\Omega} A_{t} d \mu(t)\right|^{2} d \mu(t) \in B(H)
$$

Both families $\left(A_{s}-A_{t}\right)_{(s, t) \in \Omega^{2}}$ and $\left(B_{s}-B_{t}\right)_{(s, t) \in \Omega^{2}}$ consist of commuting normal operators and by Theorem $3.2[1]$

$$
\frac{1}{2} \int_{\Omega^{2}}\left(A_{s}-A_{t}\right) X\left(B_{s}-B_{t}\right) d(\mu \times \mu)(s, t) \in \mathcal{C}_{|\| \cdot||| \mid}(\mathcal{H})
$$

due to identity (14), and so the conclusion (13) follows.

Lemma 2. Let $\mu$ (resp. $v$ ) be a probability measure on $\Omega$ (resp. $\mho$ ). Further, let both families $\left\{A_{s}, C_{t}\right\}_{(s, t) \in \Omega \times \mho}$ and

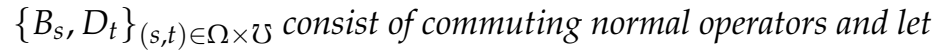


$\sqrt{\int_{\Omega}\left|A_{s}\right|^{2} d \mu(s) \int_{\mho}\left|C_{t}\right|^{2} d v(t)-\left|\int_{\Omega} A_{s} d \mu(s) \int_{\mho} C_{t} d v(t)\right|^{2}} X \sqrt{\int_{\Omega}\left|B_{s}\right|^{2} d \mu(s) \int_{\mho}\left|D_{t}\right|^{2} d v(t)-\left|\int_{\Omega} B_{s} d \mu(s) \int_{\mho} D_{t} d v(t)\right|^{2}}$ be in $\mathcal{C}_{|\| \cdot||| \mid}(\mathcal{H})$ for some $X \in \mathcal{B}(\mathcal{H})$. Then

$$
\int_{\Omega} \int_{\mho} A_{s} C_{t} X B_{s} D_{t} d \mu(s) d v(t)-\int_{\Omega} A_{s} d \mu(s) \int_{\mho} C_{t} d v(t) X \int_{\Omega} B_{s} d \mu(s) \int_{\mho} D_{t} d v(t) \in \mathcal{C}_{|\| \cdot||| \mid}(\mathcal{H}) .
$$

Proof. Apply Theorem 3 to the probability measure $\mu \times v$ on $\Omega \times \mho$ and families $\left(A_{s} C_{t}\right)_{(s, t) \in \Omega \times \mho}$ and $\left(B_{s} D_{t}\right)_{(s, t) \in \Omega \times \mho}$ of normal commuting operators in $L_{G}^{2}(\Omega \times \mho, d \mu \times v, \mathcal{B}(\mathcal{H}))$. The rest follows trivially.

Next, we consider Landau type inequality for i.p.t.i transformers in Schatten ideals for the Schatten p-norms.

Proposition 5. Let $\mu$ be a probability measure on $\Omega$ and $\left(A_{t}\right)_{t \in \Omega}$ and $\left(B_{t}\right)_{t \in \Omega}$ be $\mu$-weak ${ }^{*}$ measurable families of bounded Hilbert space operators such that $\int_{\Omega}\left(\left\|A_{t} f\right\|^{2}+\left\|A_{t}^{*} f\right\|^{2}+\left\|B_{t} f\right\|^{2}+\left\|B_{t}^{*} f\right\|^{2}\right) d \mu(t)<\infty$ for all $f \in \mathcal{H}$ and let $p, q, r \geq 1$ such that $\frac{1}{p}=\frac{1}{2 q}+\frac{1}{2 r}$. Then for all $X \in \mathcal{C}_{p}(\mathcal{H})$,

$$
\begin{aligned}
& \left\|\int_{\Omega} A_{t} X B_{t} d \mu(t)-\int_{\Omega} A_{t} d \mu(t) X \int_{\Omega} B_{t} d \mu(t)\right\|_{p} \\
& \left.\leqslant\left.\left\|\left(\int_{\Omega}\left|\left(\int_{\Omega}\left|A_{t}^{*}-\int_{\Omega} A_{t}^{*} d \mu(t)\right|^{2} d \mu(t)\right)^{\frac{q-1}{2}}\left(A_{t}-\int_{\Omega} A_{t} d \mu(t)\right)\right|^{2} d \mu(t)\right)^{\frac{1}{2 q}}\right\|^{\frac{r-1}{2}}\left(B_{t}^{*}-\int_{\Omega} B_{t}^{*} d \mu(t)\right)\right|^{2} d \mu(t)\right)^{\frac{1}{2 r}} \|_{p} .
\end{aligned}
$$

Proof. According to identity (14), applying Theorem $3.3[1]$ to families $\left(\mathcal{A}_{s}-\mathcal{A}_{t}\right)_{(s, t) \in \Omega^{2}}$ and $\left(\mathcal{B}_{s}-\mathcal{B}_{t}\right)_{(s, t) \in \Omega^{2}}$ gives

$$
\begin{aligned}
& \left\|\int_{\Omega} A_{t} X B_{t} d \mu(t)-\int_{\Omega} \mathcal{A}_{t} d \mu(t) X \int_{\Omega} \mathcal{B}_{t} d \mu(t)\right\|_{p}=\left\|\frac{1}{2} \int_{\Omega^{2}}\left(A_{s}-A_{t}\right) X\left(B_{s}-B_{t}\right) d(\mu \times \mu)(s, t)\right\|_{p} \\
& \left.\leq \|\left(\frac{1}{2} \int_{\Omega^{2}}\left(\mathcal{A}_{s}^{*}-\mathcal{A}_{t}^{*}\right)\left(\frac{1}{2} \int_{\Omega^{2}}\left|\mathcal{A}_{s}^{*}-\mathcal{A}_{t}^{*}\right|^{2}(\mu \times \mu)(s, t)\right)\right)^{q-1}\left(\mathcal{A}_{s}-\mathcal{A}_{t}\right) d(\mu \times \mu)(s, t)\right)^{\frac{1}{2 q}} \| \\
& \times\left\|\left(\frac{1}{2} \int_{\Omega^{2}}\left(\mathcal{B}_{s}-\mathcal{B}_{t}\right)\left(\frac{1}{2} \int_{\Omega^{2}}\left|\mathcal{B}_{s}-\mathcal{B}_{t}\right|^{2}(\mu \times \mu)(s, t)\right)^{r-1}\left(\mathcal{B}_{s}^{*}-\mathcal{B}_{t}^{*}\right) d(\mu \times \mu)(s, t)\right)^{\frac{1}{2 r}}\right\|_{p}
\end{aligned}
$$

By applying identity (14) once again, the last expression in (16) becomes

$$
\begin{gathered}
\|\left(\frac{1}{2} \int_{\Omega^{2}}\left(\mathcal{A}_{s}-\mathcal{A}_{t}\right)^{*}\left(\int_{\Omega}\left|\mathcal{A}_{t}^{*}-\int_{\Omega} \mathcal{A}_{t}^{*} d \mu(t)\right|^{2} d \mu(t)\right)^{q-1}\left(\mathcal{A}_{s}-\mathcal{A}_{t}\right) d(\mu \times \mu)(s, t)\right)^{\frac{1}{2 q}} \\
\left(\frac{1}{2} \int_{\Omega^{2}}\left(\mathcal{B}_{s}-\mathcal{B}_{t}\right)\left(\int_{\Omega}\left|\mathcal{B}_{s}-\int_{\Omega} \mathcal{B}_{t} d \mu(t)\right|^{2} d \mu(s)\right)^{r-1}\left(\mathcal{B}_{s}-\mathcal{B}_{t}\right)^{*} d(\mu \times \mu)(s, t)\right)^{\frac{1}{2 r}} \|_{p}
\end{gathered}
$$


Denoting $\left(\int_{\Omega}\left|A_{s}^{*}-\int_{\Omega} \mathcal{A}^{*} d \mu\right|^{2} d \mu(s)\right)^{\frac{p-1}{2}}\left(\operatorname{resp} . \quad\left(\int_{\Omega}\left|B_{s}-\int_{\Omega} \mathcal{B} d \mu\right|^{2} d \mu(s)\right)^{\frac{r-1}{2}}\right)$ by $Y$ (resp. Z), then the expression (16) becomes

$$
\left\|\left(\frac{1}{2} \int_{\Omega^{2}}\left|Y A_{s}-Y A_{t}\right|^{2} d(\mu \times \mu)(s, t)\right)^{\frac{1}{2 q}} \cdot\left(\frac{1}{2} \int_{\Omega^{2}}\left|Z B_{s}^{*}-Z B_{t}^{*}\right|^{2} d(\mu \times \mu)(s, t)\right)^{\frac{1}{2 r}}\right\|_{p} .
$$

Again applying identity (14) to families $\left(Y A_{t}\right)_{t \in \Omega}$ and $\left(Z B_{t}^{*}\right)_{t \in \Omega}$, (17) becomes

$$
\left\|\left(\int_{\Omega}\left|Y \mathcal{A}_{t}-\int_{\Omega} Y \mathcal{A}_{t} d \mu(t)\right|^{2} d \mu(t)\right)^{\frac{1}{2 q}} \cdot\left(\int_{\Omega}\left|Z \mathcal{B}_{t}^{*}-\int_{\Omega} Z \mathcal{B}_{t}^{*} d \mu(t)\right|^{2} d \mu(t)\right)^{\frac{1}{2 r}}\right\|_{p},
$$

which obviously equals to the righthand side of (15).

The next result [1] is a special case of an abstract Hölder inequality presented in Theorem 3.1.(e) [1] for Cauchy-Schwarz inequality for $0 . v$. functions in $u$.i. norm ideals.

Proposition 6. Let $\mu$ be a measure on $\Omega$. Further, let $\left(A_{t}\right)_{t \in \Omega}$ and $\left(B_{t}\right)_{t \in \Omega}$ be $\mu$-weak ${ }^{*}$ measurable in $\mathcal{B}(\mathcal{H})$ such that $\left.\left.\left|\int_{\Omega}\right| A_{t}\right|^{2} d \mu(t)\right|^{\theta}$ and $\left.\left.\left|\int_{\Omega}\right| B_{t}\right|^{2} d \mu(t)\right|^{\theta}$ are in $\mathcal{C}_{\||.|\|} \mathcal{H}$ for some $\theta>0$ and for u.i. norm. Then we have

$$
\left.\|\| \int_{\Omega} A_{t}^{*} B_{t} d \mu(t)\|\|\right|^{\theta}\|\| \leq\left.\left.\|\| \int_{\Omega} A_{t}^{*} A_{t} d \mu(t)\|\|\right|^{\theta}\|\|^{\frac{1}{2}}\|\| \int_{\Omega} B_{t}^{*} B_{t} d \mu(t)\|\|\right|^{\theta}\|\|^{\frac{1}{2}}
$$

Proof. Take $\Phi$ to be a s.g. function that generates $u$.i. norm $\|\mid \cdot\| l, \Phi_{1}=\Phi, \Phi_{2}=\Phi_{3}=\Phi^{(2)}$ (2-reconvexization of $\Phi), \alpha=2 \theta$ and $X=I$, and then apply Theorem 3.1 [1], we get our desired result.

Now, we give another generalization of Landau inequality for Gel'fand integrals of $0 . v$. functions in $u$.i. norm ideals.

Theorem 4. If $\mu$ is a probability measure on $\Omega, \theta>0$ and $\left(A_{t}\right)_{t \in \Omega}$ and $\left(B_{t}\right)_{t \in \Omega}$ are as in Proposition 6, $\mu$-weak* measurable families of bounded Hilbert space operators such that $\left.\left\||| \int_{\Omega}\left|A_{t}\right|^{2} d \mu(t)\right\|||\right|^{\theta}$ and $\left.\left\||| \int_{\Omega}\left|B_{t}\right|^{2} d \mu(t)\right\|||\right|^{\theta}$ are in $\mathcal{C}_{\||.|\|} \mathcal{H}$ for some $\theta>0$ and for some u.i. norm $\||\cdot \||$ we have

$$
\begin{aligned}
& \left.\left\|\left|\int_{\Omega} A_{t}^{*} B_{t} d \mu(t)-\int_{\Omega} A_{t}^{*} d \mu(t) \int_{\Omega} B_{t} d \mu(t)\|\|\right|^{\theta}\right\|\right|^{2} \\
& \leq\left\|\left|\int_{\Omega}\|\| A_{t}\left\|\left.\right|^{2} d \mu(t)-\right\|\left\|\int_{\Omega} A_{t} d \mu(t)\right\|\left\|\left.\right|^{2}\right\|\left\|\left.\right|^{\theta}\right\|\|\|\left\|\int_{\Omega}\right\|\left\|B_{t}\right\|\left\|\left.\right|^{2} d \mu(t)-\right\|\left\|\int_{\Omega} B_{t} d \mu(t)\right\|\left\|^{2}\right\|\left\|\left.\right|^{\theta}\right\| .\right.\right.
\end{aligned}
$$

Proof. It suffices to invoke Proposition 6 to $0 . v$. families $\left(A_{s}-A_{t}\right)_{(s, t) \in \Omega^{2}}$ and $\left(B_{s}-B_{t}\right)_{(s, t) \in \Omega^{2}}$ and use identity [7] to proof this result.

Now, we consider some interesting quantities that relate to norm inequalities. For bounded set of operators $A=\left(\mathcal{A}_{t}\right)_{t \in \Omega}$, we see that the radius of the smallest disk that essentially contains its range is

$$
r_{\infty}(A)=\inf _{A \in \mathcal{B}(\mathcal{H})} \text { ess } \sup _{t \in \Omega}\left\|A_{t}-A\right\|=\inf _{A \in \mathcal{B}(\mathcal{H})}\left\|A_{t}-A\right\|_{\infty}=\min _{A \in \mathcal{B}(\mathcal{H})}\left\|A_{t}-A\right\|_{\infty} .
$$

From the triangle inequality, we have $\left|\left\|\mathcal{A}_{t}-A^{\prime}\right\|-\left\|\mathcal{A}_{t}-A\right\|\right| \leq\left\|A^{\prime}-A\right\|$, so the mapping $A \rightarrow$ $\operatorname{ess}_{\sup } \sin _{t \in \Omega}\left\|A_{t}-A\right\|$ is nonnegative and continuous on $\mathcal{B}(\mathcal{H})$. Since $\left(\mathcal{A}_{t}\right)_{t \in \Omega}$ is bounded field of operators, we also have $\left\|A_{t}-A\right\| \rightarrow \infty$ when $\|A\| \rightarrow \infty$, so this mapping attains minimum [5], and it actually attains at some $A_{0} \in \mathcal{B}(\mathcal{H})$, which represents a center of the disk considered [6]. Any such field of operators is of finite diameter, therefore, we have that $r_{\infty}(A)=e s s \sup _{s, t \in \Omega}\left\|A_{s}-A_{t}\right\|$, with the simple inequalities given as $r_{\infty}(A) \leq \operatorname{diam}_{\infty}(A) \leq 2 r_{\infty}(A)$ relating those quantities. For such fields of operators we can now state the following stronger version of Grüss inequality [2]. 
Lemma 3. Let $\mu$ be a $\sigma$-finite measure on $\Omega$ and let $A=\left(\mathcal{A}_{t}\right)_{t \in \Omega}$ and $B=\left(\mathcal{B}_{t}\right)_{t \in \Omega}$ be $[\mu]$ a.e. bounded fields of operators. Then, for all $X \in \mathcal{C}_{|\|| \cdot||}(\mathcal{H}), \sup _{\mu(\delta)>0}\left\|\left|\frac{1}{\mu(\delta)} \int_{\delta} \mathcal{A}_{t} X \mathcal{B}_{t} d \mu(t)-\frac{1}{\mu(\delta)} \int_{\delta} \mathcal{A}_{t} d \mu(t) X \frac{1}{\mu(\delta)} \int_{\delta} \mathcal{B}_{t} d \mu(t)\right|\right\| \leq$ $\min _{i} \mathcal{P}_{i} \cdot\||X \||$. (Here sup is taken over all measurable sets $\delta \subseteq \Omega$ such that $0<\mu(\delta)<\infty$ ).

Lemma 3 has the following immediate implication when $\left(\mathcal{A}_{t}\right)_{t \in \Omega}$ and $\left(\mathcal{B}_{t}\right)_{t \in \Omega}$ are bounded fields of self-adjoint operators.

Theorem 5. If $\mu$ is a probability measure on $\Omega$ and $C, D, E, F$ be bounded self-adjoint operators. Also, let $\left(\mathcal{A}_{t}\right)_{t \in \Omega}$ and $\left(\mathcal{B}_{t}\right)_{t \in \Omega}$ be bounded self-adjoint fields satisfying $C \leq \mathcal{A}_{t} \leq D$ and $E \leq \mathcal{B}_{t} \leq F$ for all $t \in \Omega$. Then for all $X \in \mathcal{C}_{|\| .|||}(\mathcal{H})$, we have

$$
\left\|\left|\int_{\Omega} \mathcal{A}_{t} X \mathcal{B}_{t} d \mu(t)-\int_{\Omega} \mathcal{A}_{t} d \mu(t) X \int_{\Omega} \mathcal{B}_{t} d \mu(t)\right|\right\| \leq \frac{\|D-C\| \cdot\|F-E\|}{4} \cdot\||X|\| .
$$

Proof. As $\frac{C-D}{2} \leq \mathcal{A}_{t}-\frac{C+D}{2} \leq \frac{D-C}{2}$ for every $t \in \Omega$, then

$$
\text { ess sup } \operatorname{su}_{t \in \Omega}\left\|\mathcal{A}_{t}-\frac{C+D}{2}\right\|=\text { ess } \sup _{t \in \Omega} \sup _{\|f\|=1} \|\left|\left\langle\mathcal{A}_{t}-\frac{C+D}{2} \| f, f\right\rangle\right| \leq \sup _{\|f\|=1}\left|\left\langle\frac{D-C}{2} f, f\right\rangle\right|=\frac{\|D-C\|}{2}
$$

which implies $r_{\infty}(A) \leq \frac{\|D-C\|}{2}$, and similarly $r_{\infty}(B) \leq \frac{\|F-E\|}{2}$. Thus (18) follows directly.

In case of $\mathcal{H}=\mathbb{C}$ and $\mu$ being the normalized Lebesgue measure on $[a, b]$ (i.e. $d \mu(t)=\frac{d t}{b-a}$ ), then (1) follows from Theorem 5 . This special case also confirms the sharpness of the constant $\frac{1}{4}$ in the inequality (18).

Lastly, we consider, the Grüss type inequality for elementary operators in the example below.

Example 1. Let $A_{1}, \ldots, A_{n}, B_{1}, \ldots, B_{n}, C, D, E$ and $F$ be bounded linear self-adjoint operators acting on a Hilbert space $\mathcal{H}$ such that $C \leq A_{i} \leq D$ and $E \leq B_{i} \leq F$ for all $i=1,2, \cdots, n$, then for arbitrary $X \in \mathcal{C}_{\||\cdot|\|} \mathcal{H}$, we have

$$
\left\|\left|\frac{1}{n} \sum_{i=1}^{n} A_{i} X B_{i}-\frac{1}{n^{2}} \sum_{i=1}^{n} A_{i} X \sum_{i=1}^{n} B_{i}\left\|\left|\leq \frac{\|D-C\|\|F-E\|}{4}\||X \||\right.\right.\right.\right.
$$

Indeed, it is sufficient to prove that the elementary operator is normally represented and that Grüss type inequality holds for it [3].

In the next section, we dedicate our effort to the applications of this study in other fields. We consider quantum theory in particular, whereby, we describe the application in quantum chemistry and quantum mechanics.

\section{Applications in quantum theory}

Norm inequalities and other properties of i.p.t.i transformers have various applications in other fields. We discuss the applications in quantum theory involving two cases [3]. The first case is in quantum chemistry, whereby, we consider the Hamiltonian which is a bounded, self-adjoint operator on some infinite-dimensional Hilbert space which governs a quantum chemical system. The Hamiltonian helps in estimation of ground state energies of chemical systems via subsystems.

The quantum mechanics deals with commutator approximation. The discussions of approximation by commutators $A X-X A$ or by generalized commutator $A X-X B$ originates from quantum theory. For instance, the Heisenberg uncertainly principle may be mathematically deduced as saying that there exists a pair $A, X$ of linear operators and a non-zero scalar $\alpha$ for which $A X-X A=\alpha I$. A natural question immediately arises: How close can $A X-X A$ be to the identity? In [3], it is discussed that if $A$ is normal, then, for all $X \in B(H)$, $\|I-(A X-X A)\| \geq\|I\|$. In the inequality here, the zero commutator is a commutator approximate in $B(H)$.

Acknowledgments: The author is grateful to the referees for the useful comments

Conflicts of Interest: "The author declares no conflict of interest."

\section{References}


[1] Jocić, D. R. (2005). Cauchy-Schwarz norm inequalities for weak*-integrals of operator valued functions. Journal of Function Analysis, 218, 318-346.

[2] Jocić, D. R. (2009). Interpolation norms between row and column spaces and the norm problem for elementary operators. Linear Algebra Applications, 430, 2961-2974.

[3] Okelo, N. B, Agure, J. O., \& Oleche, P. O. (2013). Various notions of orthogonality in normed spaces. Acta Mathematica Scientia, 33, 1387-1397.

[4] Jocić, D. R. (1999). The Cauchy-Schwarz norm inequality for elementary operators in Schatten ideals. Journal of London Mathematical Society, 60, 925-934.

[5] Conway, J. B. (1990). A course in Functional Analysis, Second edition. Springer-Verlag, New York.

[6] Kreyzig, E. (1978). Introductory Functional Analysis with Applications. John Wiley and sons, New York.

[7] Li, X., Mohapatra, R. N., \& Rodriguez, R. S. (2002). Grüss-type inequalities. Journal of Mathematical Analysis Applications, 267, 434-443.

(C) 2020 by the authors; licensee PSRP, Lahore, Pakistan. This article is an open access article distributed under the terms and conditions of the Creative Commons Attribution (CC-BY) license (http://creativecommons.org/licenses/by/4.0/). 